\title{
10-year evaluation of the use of self-managed abortion through telemedicine: A retrospective cohort study.
}

\author{
Hanna Nortén ${ }^{1}$, Jack Wilkinson ${ }^{2}$, Onaedo Ilozumba ${ }^{1}$, Kristina Gemzell-Danielsson ${ }^{3}$, and \\ Rebecca Gomperts ${ }^{4}$
}

${ }^{1}$ Vrije Universiteit Amsterdam

${ }^{2}$ University of Manchester

${ }^{3}$ Karolinska Institutet

${ }^{4}$ Women on Web

September 16, 2020

\begin{abstract}
Objective: This study aims to provide insight into the use, acceptability and outcome of a large group of women who self-managed medical abortion. Design: Retrospective cohort study. Setting: Self-managed abortion worldwide. Population/Sample: 30344 women who completed the follow up from of the telemedical abortion service Women on Web from January 2009 till January 2020. Methods: Analyses of the evaluation forms, binary logistic regressions were performed to test the association between year and outcomes for the total sample. Main outcome measures: Demographic information including age, economic situation, country of living,knowledge about abortion pills and acceptability. Rate of complete abortions, surgical interventions, ongoing pregnancies, blood transfusions per year. Results: 30344 women from 183 different countries completed the follow-up evaluation. In total 26076 women reported taking the medical abortion pills, of which $1.5 \%$ reported an ongoing pregnancy, $10.2 \%$ a surgical intervention, and $0.6 \%$ a blood transfusion. $99 \%$ found it to be an acceptable method. $35 \%$ found it difficult to make a donation for the service and $8.5 \%$ received the service for free. $59.2 \%$ reported that they knew about medical abortion before finding Women on Web. We found a significant increase in complete abortions in 2019 (OR=1.92; 95\% CI: $1.59-2.31$ ), and decrease in surgical interventions (OR=0.49; 95\%CI: $0.40-0.60$ ) compared to 2009. Conclusion: The findings of the outcomes of the 26076 self-managed abortions in this study are in line with previous research , suggesting medical abortion through telemedicine is safe, effective and highly acceptable to women.
\end{abstract}

\section{Tweetable abstract}

Evaluation of 26076 women who self-managed abortion in restrictive settings shows high safety, effectivity and acceptability.

\section{Introduction}

Around $44 \%$ of pregnancies worldwide are unintended and approximately half of these result in induced abortion $^{(1)}$. Restrictive laws on access to abortion services do not decrease abortion rates but increases the likeliness of abortions to be unsafe ${ }^{(2)}$. Approximately 700 million women of reproductive age live in countries with restrictive abortion laws ${ }^{(3)}$. Additional barriers to access services, irrespective of legal setting, include long-waiting lists, long-distance, lack of transport, high costs and stigma ${ }^{(4)}$. Among the 56 million abortions that were estimated to take place yearly between $2010-2014,25$ million $(45 \%)$ were estimated to be unsafe ${ }^{(5)}$. In 2014 at least 22800 women worldwide died as a direct result of unsafe abortion ${ }^{(1)}$.

Medical abortion with a combination of mifepristone and a prostaglandin analogue was first developed in the 1980ies ${ }^{(6)}$. Subsequently, the method has been simplified and demedicalized while maintaining high efficacy, 
safety and acceptability. This development included home use of misoprostol, simplified follow up and task shifting to the midlevel provider and to women themselves ${ }^{(7-10)}$.

Telemedicine in terms of abortion care refers to the use of telecommunications technology by health care professionals, to provide information, counselling, and provision and clinical guidance of medical abortion pills ${ }^{(11)}$. Telemedicine has become increasingly available, both in formal and informal healthcare settings, and is a promising solution to make abortion services more accessible, particularly for those living in rural areas and legally restricted settings ${ }^{(12,13)}$. Studies of the use of medical abortion in formal settings have found it to be non-inferior to in-clinic provision of services ${ }^{(14-16)}$. Similarly, emerging evidence is showing that medical abortion through telemedicine outside formal healthcare settings is safe, effective and an acceptable method for women ${ }^{(11)}$.

However, gaps remain in evidence on self-managed abortion through telemedicine, and a comprehensive overview of trends in the usage is lacking (17). Two recent reviews on self-managed abortion identified critical areas of future research, including further evidence on physical, social and emotional experiences, and that study settings include diversity in legal restrictions, geographic and social context ${ }^{(12,17)}$. Therefore, this study aims to provide insight into the use and outcome of self-managed medical abortion through Women on Web, a telemedical abortion service which is operating worldwide, by evaluating follow-up data of women's self-managed abortion experiences over the past ten years.

\section{Methods}

\section{Study Design}

This study was a retrospective cohort study, using a quantitative approach to evaluate the changes over time in the use and outcome of medical abortion by analysing the data of Women on Web.

\section{Study setting}

Women on Web (WoW) is a non-profit organization, providing telemedicine services since 2006. Services include the provision of information, medical guidance and medical abortion for women living in countries with restricted access to safe abortion ${ }^{(18)}$. WoW mainly provide their services through their website ${ }^{(18)}$. Women opting for a medical abortion complete an online consultation with a medical doctor. If they are less than 10 weeks pregnant and have no contradictions, they can be sent abortion pills with detailed instructions. WoW requests a donation of between 70-90 euros, but a lower donation can be made or the service is provided for free in case of financial hardship.

\section{Study population}

A total of 81683 women were sent medical abortion medication between 2009-2019. To be included in the main data analysis, women had to report that they used the medical abortion medication and complete the follow-up evaluation. Women who did not go through with the abortion and completely unanswered surveys were excluded for data analysis.

\section{Data collection}

Data including the consultation and follow-up survey was self-reported into the WoW website. Data was transported to an Excel file and anonymized. Follow-up surveys were sent via e-mail five weeks after the shipment of medication. If needed, a reminder was sent after another two weeks. Similar to follow-up instruments in clinical settings, the survey consisted of 30 questions which included information on completed treatment, how the pregnancy was confirmed, perceived side-effects, any visits or contacts for medical care, any treatment received, how information was accessed and emotions after the procedure. Although WoW has been active since 2006, we included data from the year 2009 onwards as this was the year WoW changed its data collection system.

\section{Measurements}


Outcomes included: numbers and rates of women who self-managed medical abortion, their age, socioeconomic situation, country of living, knowledge on medical abortion, rate of reported ongoing pregnancy, surgical intervention, complete abortion, blood transfusion, if they found it acceptable to receive telemedical abortion service method, if the abortion pill was the appropriate method and if they would recommend the service to a friend ${ }^{(11,12)}$. Ongoing pregnancy is defined as the failure of medical abortion ${ }^{(19)}$. The Medical Abortion Reporting Efficacy (MARE) guidelines recommend defining a medical abortion to be successful when there is a "successful expulsion of the intrauterine pregnancy without the need for surgical intervention", i.e. a complete abortion ${ }^{(19)}$. Lastly, a blood transfusion is an adverse event which is often measured as an indication of safety ${ }^{(17)}$.

Countries were initially divided into UN geographic regions and sub-regions ${ }^{(20)}$. As the frequencies of evaluations and representation of countries showed to be extremely varied, it was concluded to be a misleading classification. Thus, only the top 10 most frequent countries were included for an overview, as they accounted for $84,3 \%$ of the total sample, and $84,9 \%$ after inclusion criteria.

\section{Statistical analysis}

Data was cleaned using Microsoft Excel and further analysed in IBM SPSS 26. Invalid or missing answers were corrected or deleted by evaluating women's comments or subsequent responses throughout the evaluation. If contradictory information was provided in the evaluation form, e-mail correspondence was checked in the WoW database for the original answer. Descriptive statistical analysis was performed on demographic information and main outcomes. For questions with multiple answers, new categories were created, and variables were re-coded into binary variables in accordance with previous studies $(11,16,19,21,22) .7$ age categories were created; <20, 20-24, 25-29, 30-34, 35-39, 40-44, [?]45 based on previous literature and for the purposes of summarising the data, but age was included as a continuous variable in logistic regression analysis $^{(21)}$. Evaluations from Ireland and Northern Ireland were grouped, in accordance with previous research using WoW data ${ }^{(21)}$. A binary logistic regression analysis was performed to test associations further. Four regressions were run for the total sample and year, adjusted for age. The year 2009 was set as the reference category.

\section{Results}

Abortion pills were provided to 81683 women between 2009-2019. A total of 30344 (37.2\%) women from 183 different countries completed the follow-up evaluation between January 2009 and December 2019, of which $26076(85.6 \%)$ reported that they used the abortion pills and were included for main data analysis. 3780 $(12.4 \%)$ women did not use the abortion pills, and $488(1.6 \%)$ evaluations were completely unanswered. Reasons for not completing the medical abortion included: deciding to continue the pregnancy $(\mathrm{n}=737)$, having a spontaneous miscarriage meanwhile $(\mathrm{n}=1839)$, deciding to use another method meanwhile $(\mathrm{n}=1040)$, realising they were not pregnant $(\mathrm{n}=39)$, having issues with customs $(\mathrm{n}=121)$ and having an ectopic pregnancy $(\mathrm{n}=4)$. Missing data was relatively low, ranging from $0.5-0.7 \%$ and was deleted from the subsequent regression analysis.

(Table 1. Overview of study population.)

A summary of the main outcome measures is presented in Table 2. Ongoing pregnancy was reported by 404 $(1.5 \%)$ women while $22926(87.9 \%)$ reported a complete abortion. The overall rate of blood transfusion was $0.6 \%(\mathrm{n}=155)$. As a proxy of economic situation, we found that $9175(35 \%)$ who made a donation for the service reported that it was difficult to find the money . In $8.5 \%$ of cases $(n=2223)$ the service was fully donated. Just over half of the women reported prior knowledge of medical abortion. 96.6\% ( $\mathrm{n}=25178)$ found medical abortion to be an appropriate method for them, and 99\% (25186) found it to be acceptable of having the medical abortion at home with WoW support. 96.1\% ( $\mathrm{n}=25071)$ would recommend WoW services to other women in a similar situation.

(Table 2. Frequency table of use and outcome of medical abortion.)

Results from logistic regression analysis are summarised in Table 3. Year of treatment was significantly 
associated with all outcomes, except for knowledge about medical abortion. Models were adjusted for age, which had an OR and CI close or equal to 1, indicating no association for each outcome. Overall, the chance of having a complete abortion increased with year, OR 1.92 (95\% CI: $1.59-2.31) 2019$ versus 2009. The risk of having surgical intervention significantly decreased from 2012 onwards, with an OR of 0.49 (95\% CI: $0.40-0.60)$ in 2019 versus 2009.

(Table 3. Changes in the use and outcome of medical abortions between 2009-2019.)

\section{Discussion}

\section{Main Findings}

This large study including 30344 women from 183 countries using telemedical medical abortion identified several changes in the use and outcome of medical abortions over the 10 years of evaluation. A significant association was found between the year of treatment and outcome measures. There was a significant increase in reported complete abortions, a decrease in surgical intervention, and an overall low rate of ongoing pregnancy and blood transfusions. The service was highly acceptable and medical abortion was considered an appropriate method in almost all cases.

There were significant fluctuations in financial hardship but these did not show a specific trend. There was no significant change in knowledge about medical abortion over the years.

\section{Strengths and limitations}

A key limitation is the low response rate, which appeared to decrease over time. If the likelihood of responding was related to the patient's experience and outcome, then this would be a source of bias in our results. A loss to follow-up is to be expected and is a persistent challenge within abortion research given the high level of stigma associated and the legally restricted settings ${ }^{(8,23)}$. Other limitations include the use of self-reported data. However, given that the sample was overwhelmingly in legally restricted settings and in an informal healthcare setting, using self-reported data is the most feasible method ${ }^{(21)}$. We could not adjust for known confounders, such as gestational age, as this information was not available in this dataset ${ }^{(22)}$. However, as WoW limits the availability of the service to women who are less than 9-10 weeks gestation, the the abortions in this dataset are expected to have taken place in the first trimester. To our best knowledge, this study provides the largest evaluation of the outcome of self-managed abortions provided by telemedicine. Furthermore, it includes the largest sample of women in legally restricted settings. Outcomes are reported in consistency with prior studies for comparability. In addition, we included outcome measures which have never been looked at before such as prior knowledge about medical abortion.

\section{Interpretation}

As illustrated in Table 1, all of the UN main geographic regions were represented, with a highly variable frequency of countries. When WoW was launched in 2006, it was available in Polish, English, Spanish, French and Portuguese, whereas today it is available in 24 languages ${ }^{(8,18)}$. The age distribution for the total sample is as expected and in line with previous reports, showing the peak for having an abortion is often around 20-24, but also common in the early thirties in many countries ${ }^{(24)}$.

The proportion of the total sample reporting that they knew about medical abortion before using the service was $59.2 \%$ and remained almost unchanged between 2009 to 2019. Given the advancements over the past years use and acceptability among health care providers, the WHO and NGOs of WoW services, these results suggest that many women only learn about medical abortion once they have an unwanted pregnancy, which has also been suggested previously ${ }^{(25)}$.

Our findings in regards to physical outcomes are in line with previous literature. A recent systematic review of outcomes of medical abortion through telemedicine reported a range of $0-1.9 \%$ for continuing pregnancy, 93.8-96.4\% for complete abortion, 0.9-19.3\% for surgical intervention and 0-0.7\% for blood transfusion ${ }^{(11)}$. These results are well in line with our findings except for a slightly lower rate of complete abortion of $87.9 \%$. This is still in line with rates reported in studies on in-clinic medical abortion treatment ${ }^{(16)}$. The higher 
rate in surgical interventions compared with other studies might be explained by the fact that the number of women living in Poland accounted for almost half of the total sample and the surgical intervention rate was highest in Poland (13.2\%) and Brazil (17.2\%). High intervention rates in Poland and Brazil has also been reported in previous studies ${ }^{(26-28)}$. It has been suggested that variation in surgical intervention rates are due to differences in local clinical practices and economic incentives, rather than treatment efficacy or gestational length ${ }^{(28)}$. We found an overall increase in the rate of complete abortion and decrease in surgical interventions between 2009 - 2019, which may be due to a combination of factors. Such as a rise of medical treatment of incomplete abortion, such as repeat doses of misoprostol, improved training of health professionals and increased knowledge about medical abortion process by women might have resulted in the decrease of surgical interventions ${ }^{(29)}$.

Ultimately, further research is required to establish a more comprehensive understanding of contextual differences between countries to improve services and make telemedical abortions more accessible to women.

\section{Conclusion}

The purpose of the current study was to evaluate the use, outcome and acceptability in the telemedical provision of medical abortion over the past 10 years. We identified several promising trends over the past ten years: increasing rates of complete abortion and decrease in surgical interventions. As indicated by has a high rate of complete abortion, high acceptability, low rate of ongoing pregnancy and complications such as bleeding needing a blood transfusion, the study adds to the growing body of research suggesting online telemedical counselling and provision and self-managed medical abortion is a safe, effective and highly acceptable method. While being of exploratory nature, findings suggest that there is an unmet need for information about medical abortion provided by telemedicine.

\section{Acknowledgements}

None.

\section{Disclosure of Interests}

HN and IO have none to declare. JW is supported by a Wellcome Institutional Strategic Support Fund award $[204796 / Z / 16 / Z]$ and declares that publishing research benefits his career. KGD has served as an ad hoc speaker and/ or member of advisory boards for Exelgyn, Campus Pharma and HRA Pharma. RG is the director of Women on Web.

\section{Contribution to Authorship}

HN was responsible for planning, carrying out, analysing and writing up the work. JW was responsible for analysing and editing. OI was responsible for planning and editing. KDG was responsible for conception and editing. RG was responsible for the conception, planning and writing up of the work.

\section{Details of Ethics Approval}

Ethical approval was given by Karolinska Institute on 27-1-2010. During the original data collection, participants gave informed consent to their data being used for research purposes. All data was completely anonymised.

\section{Funding}

No funding was received for this study.

\section{Keywords}

self-managed abortion, abortion pills, medical abortion, telemedical abortion, mifepristone, misoprostol

\section{References}

1. Sedgh G, Bearak J, Singh S, Bankole A, Popinchalk A, Ganatra B, et al. Abortion incidence between 1990 and 2014: global, regional, and subregional levels and trends. The Lancet. 2016;388(10041):258-67. 
2. Starrs AM, Ezeh AC, Barker G, Basu A, Bertrand JT, Blum R, et al. Accelerate progress - sexual and reproductive health and rights for all: report of the Guttmacher-Lancet Commission. The Lancet. 2018;391(10140):2642-92.

3. Center for Reproductive Rights. The World's Abortion Laws 2020 [Available from: https://reproductiverights.org/worldabortionlaws.

4. Chemlal S, Russo G. Why do they take the risk? A systematic review of the qualitative literature on informal sector abortions in settings where abortion is legal. BMC Women's Health. 2019;19(1):55.

5. Ganatra B, Gerdts C, Rossier C, Johnson BR, Jr., Tunçalp Ö, Assifi A, et al. Global, regional, and subregional classification of abortions by safety, 2010-14: estimates from a Bayesian hierarchical model. The Lancet. 2017;390(10110):2372-81.

6. Fiala C, Danielsson K-G. Review of medical abortion using mifepristone in combination with a prostaglandin analogue. Contraception. 2006;74(1):66-86.

7. Fiala C, Winikoff B, Helström L, Hellborg M, Gemzell-Danielsson K. Acceptability of home-use of misoprostol in medical abortion. Contraception. 2004;70(5):387-92.

8. Gomperts R, Jelinska K, Davies S, Gemzell-Danielsson K, Kleiverda G. Using telemedicine for termination of pregnancy with mifepristone and misoprostol in settings where there is no access to safe services. BJOG: An International Journal of Obstetrics \& Gynaecology. 2008;115(9):1171-8.

9. Oppegaard KS, Qvigstad E, Fiala C, Heikinheimo O, Benson L, Gemzell-Danielsson K. Clinical follow-up compared with self-assessment of outcome after medical abortion: a multicentre, non-inferiority, randomised, controlled trial. The Lancet. 2015;385(9969):698-704.

10. Kopp Kallner H, Fiala C, Stephansson O, Gemzell-Danielsson K. Home self-administration of vaginal misoprostol for medical abortion at 50-63 days compared with gestation of below 50 days. Human Reproduction. 2010;25(5):1153-7.

11. Endler M, Lavelanet A, Cleeve A, Ganatra B, Gomperts R, Gemzell-Danielsson K. Telemedicine for medical abortion: a systematic review. BJOG: An International Journal of Obstetrics \& Gynaecology. 2019;126(9):1094-102.

12. Endler M, Cleeve A, Gemzell-Danielsson K. Online access to abortion medications: a review of utilization and clinical outcomes. Best practice \& research Clinical obstetrics \& gynaecology. 2020;63:74-86.

13. Yang YT, Kozhimannil KB. Medication Abortion Through Telemedicine: Implications of a Ruling by the Iowa Supreme Court. Obstetrics and gynecology. 2016;127(2):313-6.

14. Baiju N, Acharya G, D’Antonio F, Berg R. Effectiveness, safety and acceptability of self-assessment of the outcome of first-trimester medical abortion: a systematic review and meta-analysis. BJOG: An International Journal of Obstetrics \& Gynaecology. 2019;126(13):1536-44.

15. Grossman D, Grindlay K. Safety of Medical Abortion Provided Through Telemedicine Compared With In Person. Obstetrics \& Gynecology. 2017;130(4).

16. Ngo TD, Park MH, Shakur H, Free C. Comparative effectiveness, safety and acceptability of medical abortion at home and in a clinic: a systematic review. Bulletin of the World Health Organization. 2011;89(5):360-70.

17. Moseson H, Herold S, Filippa S, Barr-Walker J, Baum SE, Gerdts C. Self-managed abortion: A systematic scoping review. Best practice \& research Clinical obstetrics \& gynaecology. 2020;63:87-110.

18. About Women on Web: Women on Web; [Available from: https://www.womenonweb.org/en/page/521/aboutwomen-on-web. . 
19. Creinin MD, Chen MJ. Medical abortion reporting of efficacy: the MARE guidelines. Contraception. 2016;94(2):97-103.

20. Standard country or area codes for statistical use (M49): UN Statistics Division; [Available from: https://unstats.un.org/unsd/methodology/m49/.

21. Aiken ARA, Digol I, Trussell J, Gomperts R. Self reported outcomes and adverse events after medical abortion through online telemedicine: population based study in the Republic of Ireland and Northern Ireland. BMJ. 2017;357:j2011.

22. Sjöström S, Dragoman M, Fønhus MS, Ganatra B, Gemzell-Danielsson K. Effectiveness, safety, and acceptability of first-trimester medical termination of pregnancy performed by non-doctor providers: a systematic review. BJOG: An International Journal of Obstetrics \& Gynaecology. 2017;124(13):1928-40.

23. Cameron S. Recent advances in improving the effectiveness and reducing the complications of abortion. F1000Research. 2018;7.

24. Singh S, Remez L, Sedgh G, Kwok L, Onda T. Abortion Worldwide 2017: Uneven Progress and Unequal Access. New York: Guttmacher Institute; 2018.

25. Zamberlin N, Romero M, Ramos S. Latin American women's experiences with medical abortion in settings where abortion is legally restricted. Reproductive Health. 2012;9(1):34.

26. Gomperts R, van der Vleuten K, Jelinska K, da Costa CV, Gemzell-Danielsson K, Kleiverda G. Provision of medical abortion using telemedicine in Brazil. Contraception. 2014;89(2):129-33.

27. Endler M, Beets L, Gemzell Danielsson K, Gomperts R. Safety and acceptability of medical abortion through telemedicine after 9 weeks of gestation: a population-based cohort study. BJOG: An International Journal of Obstetrics \& Gynaecology. 2019;126(5):609-18.

28. Gomperts R, Petow SAM, Jelinska K, Steen L, Gemzell-Danielsson K, Kleiverda G. Regional differences in surgical intervention following medical termination of pregnancy provided by telemedicine. Acta Obstetricia et Gynecologica Scandinavica. 2012;91(2):226-31.

29. Fiala C, Cameron S, Bombas T, Parachini M, Agostini A, Lertxundi R, et al. Outcome of first trimester medical termination of pregnancy: definitions and management. The European Journal of Contraception \& Reproductive Health Care. 2018;23(6):451-7.

\section{Hosted file}

Table 1.docx available at https://authorea.com/users/359267/articles/481388-10-yearevaluation-of-the-use-of-self-managed-abortion-through-telemedicine-a-retrospectivecohort-study

\section{Hosted file}

Table 2.docx available at https://authorea.com/users/359267/articles/481388-10-yearevaluation-of-the-use-of-self-managed-abortion-through-telemedicine-a-retrospectivecohort-study

\section{Hosted file}

Table 3.docx available at https://authorea.com/users/359267/articles/481388-10-yearevaluation-of-the-use-of-self-managed-abortion-through-telemedicine-a-retrospectivecohort-study 